\title{
EL APRENDIZAJE HISTÓRICO EN LA EDUCACIÓN SECUNDARIA. JÓVENES CHILENOS Y CONCIENCIA HISTÓRICA*
}

\author{
HISTORICAL LEARNING IN SECONDARY EDUCATION. \\ CHILEAN YOUNG AND HISTORICAL CONSCIOUSNESS
}

\author{
Fabián González Calderón**, Camila Gárate Guerrero**
}

\begin{abstract}
Este trabajo analiza algunos resultados de un estudio exploratorio realizado en cinco escuelas de la ciudad de Santiago de Chile. Las perspectivas de los jóvenes acerca de diversos temas vinculados a la historia se recogieron mediante un cuestionario cerrado de 44 preguntas que respondieron 180 estudiantes de segundo año de Educación Media. La muestra incluye colegios públicos y privados de diversas comunas de la Región Metropolitana. El artículo se concentra específicamente en los resultados obtenidos considerando las primeras seis preguntas del cuestionario, estas preguntas se referían a las formas que adopta el aprendizaje histórico en el contexto escolar. Los resultados de la investigación nos muestran la alta valoración que otorgan los jóvenes al estudio de la historia, el reconocimiento de la sólida vinculación existente entre los problemas históricos y su propia vida, todo lo anterior junto con una notoria presencia de prácticas y formas de aprendizaje de tipo tradicional. La perspectiva teórica desde la que se analizan los resultados releva categorías como aprendizaje histórico, narrativa y conciencia histórica, concibiendo el aprendizaje de la historia como una síntesis de experiencias que superan la formación escolar.
\end{abstract}

Palabras claves: Aprendizaje histórico, educación histórica, conciencia histórica, educación media.

This paper discusses some results of an exploratory study in five schools in the city of Santiago de Chile. The prospects of young people on various topics related to history were collected through a survey of 44 questions, 180 sophomores in High School participated in the study. The sample includes public and private schools in different districts of the Region Metropolitana. The article is specifically focused on the results obtained in the first six questions in the questionnaire, these questions were related to the forms adopted by historical learning in the school context. The most relevant results show the high value that give young people the study of history, recognition of a strong link between the historical problems and their practical life, all this with a strong presence of practices and forms of learning traditional type. The results were analyzed from a theoretical perspective which emphasizes concepts such as historical learning, narrative and historical consciousness conceiving learning history as a synthesis of experiences that go beyond school education.

Key words: Historical learning, historical education, historical consciousness, secondary education.

\section{Introducción}

Los jóvenes del siglo XXI se han hecho habitantes del nuevo milenio aprendiendo a respirar el aire denso de las crisis políticas y sociales que viven nuestras frágiles democracias. Han debido soportar la vertiginosa e imperceptible mutación de la vida social derivada de las nanotecnologías y las redes, experimentando una verdadera metamorfosis generacional en el contexto de la era de la globalización (Feixa 2006). Sus nuevos modos de actuación histórica son el resultado de esos profundos cambios que vienen ocurriendo en la sociedad occidental. Por cierto, estas transformaciones han afectado directamente a las instituciones y a los procesos de socialización de las nuevas generaciones, interfiriendo en la producción social de los individuos, en sus espacios y tiempos (Dayrell 2007). Como lo ha señalado Martín-Barbero (1998), lo que hay de nuevo en las sensibilidades juveniles es la percepción desconcertante de una reorganización profunda de esos modelos de socialización. Algunos autores, incluso, consideran que es preciso evaluar si, en el actual proceso de cambio histórico, los sistemas educativos están preparados para capacitar a las nuevas generaciones "en la resolución de las complejas ecuaciones históricas que les presenta su tiempo" (Salazar 2003: 322). Desde otra perspectiva, este mismo conjunto de problemáticas podría ser planteado del siguiente modo:

\footnotetext{
* Resultado del Núcleo Temático de Investigación (NTI) Memoria Crítica-UAHC 2014.

** Universidad Academia de Humanismo Cristiano, Santiago, Chile. Correos electrónicos: fgonzalez@academia.cl; cpgg@live.com
} 
La incapacidad del sistema educativo del Estado para ofrecer y garantizar educación para todos, el crecimiento del desempleo y de la sobrevivencia por medio de la economía informal, indican que el marco que sirvió como delimitación para el mundo juvenil, por la pertenencia a las instituciones educativas y a la incorporación tardía a la población económicamente activa, está en crisis (Reguillo 2000:27).

La condición juvenil expresa, entonces, una forma propia de vivir el tiempo que reconoce la indeterminación del futuro, cuestión que justificaría luchar y vivir en la inmediatez de la experiencia (Giroux 1994). Para nuestros jóvenes, el mundo se encuentra anclado en el presente. Las identidades juveniles se construyen a partir de la misma trama difusa que caracteriza a la historicidad del presente, un tiempo donde la pérdida de sentido derivada de la incoherencia de los sistemas de referencia provoca que los territorios juveniles se enmarquen entre los límites que fija el escapismo y la evasión, donde la violencia social o la droga, la rebelión y el delito son parte del paisaje diario (Salazar 2003; Citron 1982). Este permanente vértigo con el que se vive el presente por los jóvenes ha sido astutamente captado por el mercado, mientras que la desaparición de expectativas se ha convertido en el futuro de vida de los jóvenes (Giroux 1994; Reguillo 2000). Garantizar la propia sobrevivencia es el más cotidiano de los desafíos juveniles, en una tensión constante entre la búsqueda de gratificación inmediata y un posible proyecto de futuro; la identidad, que se forja en ese intersticio, basada en la expectativa de llegar a ser alguien, es, finalmente, una construcción del presente (Dayrell 2007; Sposito 1999). Por todo lo anterior, comprender las condiciones cambiantes de la formación de la identidad en las culturas mediadas electrónicamente será, según H. Giroux (1994), una cuestión extraordinariamente útil para los educadores críticos.

De cualquier modo, los jóvenes pueden ser vistos como un grupo emergente que se ve obligado a competir por la conquista de un mismo espacio social, donde la resistencia y el conflicto entre las generaciones hacen posibles nuevas alternativas visibilizando la idea de cambio (Sposito 1999). Por lo mismo, hay quienes han enfatizado la idea de ruptura como clave para entender la nueva relación cultural de los jóvenes con las generaciones precedentes (Martín-Barbero 1998). Este concepto lejos de expresar empantanamiento o inmovilidad permite descifrar el desplazamiento que las nuevas generaciones realizan en el tiempo.

Así, diversos y desiguales, los jóvenes siguen señalando, mediante múltiples modos, su disconformidad (y discontinuidad) con todos aquellos proyectos sociales que sembraron promesas en los campos de un futuro que nunca llegó (Reguillo 2000). Cotidianamente infantilizados y prisioneros de un futuro laboral incierto aparecen, al decir de R. Cuesta (1998), condenados a ser felices gracias al consumo de bienes efímeros. Y por añadidura, desarrollan una actitud compleja y extraordinariamente contradictoria en relación con la enseñanza de la historia.

\section{Jóvenes chilenos y conciencia histórica}

El historiador Gabriel Salazar (2003) graficó las complejidades que cruzan e impactan la realidad juvenil chilena señalando que, sobre ella, permanentemente llueven anacronismos. Precisamente, fue a partir de la década de los 90 del siglo pasado, en medio de un clima social contradictorio y desconcertante, que los jóvenes chilenos se vieron forzados a recurrir a lo que Salazar denominó intuición histórica, cuestión que, por cierto, no impidió que por esos años se elevaran radicalmente los niveles de enajenación y desconexión juvenil. Una vez finalizado el potente silencio de los 90 aquellas generaciones nacidas una vez terminada la dictadura cívico-militar se enfrentaron a una sociedad bastante diferente de aquella que vio crecer y envejecer a sus padres y abuelos (Salazar y Pinto 2002). El joven urbano popular, por ejemplo, se ve obligado a resolver la inestabilidad histórica que le ofrece la sociedad neoliberal "seleccionando las ofertas que el sistema en transformación dispone para ellos. En ese sentido, construyen un collage con las ofertas sociales del sistema; de ahí el lugar común de estar salvando, que refleja su particular sentido estratégico para abordar la existencia" (Zarzuri \& Ganter 2002).

De acuerdo con Cuesta (1998), como resultado de este tipo de procesos socioculturales, y de algunas otras transformaciones de mayor envergadura comunes a las sociedades contemporáneas, la conciencia histórica de los jóvenes tiende a ser limitada, corta e impuesta. Esto significa que su memoria:

"carece de los asideros sociales y culturales que permiten interiorizar las formas 
académicas del saber histórico. La cultura histórica dominante en los centros de secundaria choca con una buena parte de los supuestos destinatarios de la educación histórica de la era de la escolarización de masas. Colisiona con la cultura y el lenguaje de las clases sociales subalternas, con las experiencias vitales fragmentarias de la mayoría de los alumnos e incluso con los valores dominantes en la vida social" (Cuesta 1998:176).

Ahora bien, es preciso anotar que en Chile, a partir de los primeros años del nuevo milenio, distintos modos de expresión fueron anunciando, con distintas intensidades, la acumulación de inconformidades y el desfase que habían provocado en el mundo social y juvenil los largos años de funcionamiento del modelo heredado de la dictadura (González, 2012). Otros conflictos y una violencia de nuevo tipo se fueron instalando como escenario histórico donde, poco a poco, fue fraguándose la identidad de los nuevos movimientos sociales juveniles que despertarán apenas despunte el siglo XXI (Garcés 2012). Se trata de la emergencia de nuevas experiencias de organización estudiantil, irrupciones juveniles multitudinarias en el espacio público, horizontalidad y participación sin parangón en las últimas dos décadas. La idea del joven apolítico y desinteresado comenzó a resquebrajarse rápidamente. Desde entonces, los estereotipos respecto de lo que significa ser joven en Chile han cambiado de modo significativo. Tal vez sea esta apertura de expectativas la que empuje a ciertos autores a visibilizar en la dinámica del cambio histórico una posibilidad para el aprendizaje de la historia, incluso más allá de la gramática restringida del espacio escolar:

"Es evidente que en cada hecho, en cada época y para cada generación, se abren diversos futuros, por lo tanto, también en nuestro tiempo tenemos ante nosotros diferentes futuros sobre los que debemos decidir. Esto también es una enseñanza de la Historia" (Santisteban y Anguera 2014: 251).

Esas transformaciones y emergencias que expanden el mundo juvenil señalándole nuevas fronteras pueden tener una vinculación extraordinariamente importante en el desarrollo de la conciencia histórica, especialmente si pensamos que dicha conciencia no es producto exclusivo del repertorio histórico que ofrecen las aulas y la escolarización formal. Como se ha señalado, la conciencia histórica de profesores y alumnos no se genera únicamente por sus experiencias como consumidores de historia o como sujeto u objetos del aprendizaje histórico en situación formal-escolar. Lo más probable es que dicha conciencia sea una combinación entre esa historia enseñada y aquella aprendida o socializada mediante los mass media u otros espacios sociales (Cuesta 1998). Dicho de otro modo, la familiaridad que los estudiantes desarrollan con la historia deriva de variadas fuentes, "que incluyen pero no se limitan, al currículum escolar" (Barton 2010:103). Entonces, como lo propone Bergmann (1990), resulta fundamental incluir dentro de la investigación didáctica las recepciones extraescolares de la historia. De esa forma, no solo se tematiza la historia regulada y disciplinada por la ciencia y por la enseñanza sino que también se incluye la historia transmitida en el proceso de socialización. Finalmente, se trata de ir más allá de las advertencias programáticas, más allá de los consensos editoriales, y más allá de la renovación de materiales y recursos, para indagar en torno a los saberes históricos apropiados (Pagés 2015), es decir, aquellos saberes que construyen los estudiantes en su aprendizaje y que pueden utilizar en la escuela o en su vida cotidiana. Siguiendo a J. Pagés (2015), la bitácora de la investigación didáctica en ciencias sociales debiera orientarse tras propósitos tan evidentes como, por ejemplo, qué saben de historia y de qué historia se han apropiado los estudiantes al terminar cada ciclo escolar, cómo aprendieron esa historia y cómo utilizan lo aprendido para ubicarse en el mundo.

Este tipo de investigaciones se vuelven cada vez más necesarias debido a que nos permitirían entender las ideas que estructuran las relaciones de los alumnos con el pasado y los tipos de pasado a los que han tenido acceso (Lee 2006). Por tanto, conocer las narrativas históricas que los alumnos van construyendo a los largo de su educación histórica (formal e informal) constituye una actitud investigativa esencial para comprender la construcción de la conciencia histórica juvenil (Barca 2013). Aun más, si pensamos en las metas educativas que debiera trazarse la enseñanza de la historia podríamos coincidir con Santisteban y Anguera cuando señalan que la formación de la conciencia histórica como eje de la conciencia ciudadana, 
de la conciencia crítica y de la construcción de la identidad constituiría "una gran revolución cultural, histórica y educativa de nuestro tiempo" (Santisteban y Anguera 2014:254).

La conciencia histórica, según J. Rusen (2010), funciona como un modo de orientación específico en situaciones de la vida real en el presente: con el propósito de ayudarnos en la comprensión de la realidad pasada con el fin de aprehender la realidad actual. Los presupuestos de Rüsen han sido considerados como referenciales para las concepciones de aprendizaje que orientan propuestas curriculares de historia en la sociedad contemporánea. Según Schmidt y Cainelli (2015), lo anterior incluiría también una cuestión innegociable: cualquier aprendizaje es autoeducación y es inseparable de la práctica significativa de la autogestión en que los jóvenes y niños son agentes activos de su propia educación.

Esta concepción de la conciencia histórica permite responder mejor a las necesidades de orientación temporal de un tiempo plagado de cambios vertiginosos y de experiencias temporales desconcertantes (Barca 2013; Rüsen 2010). Afrontar las concepciones de sentido común referidas a la historia por este tipo de estudios contribuirá a que sea un poco más difícil para los estudiantes de los distintos niveles educativos aceptar versiones simplificadas del pasado, descartando pronta e informadamente aproximaciones engañosas o estereotipadas (Lee 2006).

\section{Consideraciones metodológicas}

El estudio que aquí se analiza formó parte de un proyecto mayor titulado "Jóvenes y la Historia en el MERCOSUR" iniciado en 2010, a partir de estudios preliminares y un proyecto piloto realizado entre 2006 y 2009 por un equipo de investigadores de Brasil, Argentina y Uruguay (Cerri 2011). Se trata de un levantamiento de datos intercultural, basado en el proyecto europeo "Youth and History", desarrollado a mediados de los años 1990 por la Conferencia Permanente de Asociaciones de Profesores de Historia de Europa (EUROCLIO). A partir de esas experiencias internacionales, los objetivos generales que guiaron nuestra investigación fueron: conocer las características del aprendizaje histórico en el mundo escolar y su vinculación con la formación de la conciencia histórica desde la perspectiva de los estudiantes de enseñanza media de la ciudad de Santiago; y, analizar las narrativas históricas que elaboran los estudiantes de enseñanza media en torno a su experiencia en el tiempo y su actuación en el presente.

El instrumento utilizando en la investigación es un cuestionario extenso aplicado a jóvenes (y otro a sus profesores), con la mayor parte de las preguntas con respuesta cerrada basada en escala Likert. Mediante 44 preguntas, el cuestionario aborda los temas del aprendizaje histórico escolar, elementos de cultura política y de cultura histórica de los alumnos y profesores, así como situaciones simuladas de decisión que envuelven cuestiones de actualidad en las que los alumnos y profesores deben posicionarse, movilizando sus saberes, identidades y expectativas temporales. El proyecto logró recoger 3.913 cuestionarios de estudiantes de Brasil, Argentina, Uruguay, Paraguay y Chile, con 182 cuestionarios de estudiantes chilenos. La muestra elaborada es no probabilística, es decir, no es estadísticamente representativa, pero ha sido construida de acuerdo con un diseño que permite conclusiones significativas por el tamaño de la muestra. Los datos referidos a Chile fueron recogidos en cinco escuelas de la ciudad de Santiago (capital nacional y regional) y consideró 1 Escuela Pública de excelencia, 1 Escuela Pública de la periferia, 1 Escuela Pública rural, 1 Escuela Privada laica empresarial, 1 Escuela Privada confesional. Los jóvenes chilenos que respondieron el cuestionario fueron, en todos los casos, estudiantes de $2^{\circ}$ año de Educación Media, es decir, jóvenes que promedian los 15 o 16 años. El trabajo de campo se realizó entre agosto de 2012 y mayo de 2013, las encuestas fueron aplicadas en la misma sala de clases, este proceso estuvo a cargo de encuestadores debidamente capacitados.

En específico este artículo utiliza los datos correspondientes a las seis primeras preguntas $\left(\mathrm{N}^{\circ} 1\right.$ a la $\mathrm{N}^{\circ}$ 6) del instrumento destinado a los alumnos, que trataban básicamente de recoger las percepciones que tienen los estudiantes de Educación Media respecto de lo que pasa habitualmente en la clase de historia en cada uno de sus colegios. Por tanto, los datos debieran darnos luces acerca de quiénes protagonizan los procesos de aprendizaje de la historia en las aulas, qué actividades son más frecuentes o deseadas por los estudiantes, qué materiales reciben mayor o menor aprobación y, en suma, conocer cuál es la valoración general que se le otorga a este conocimiento escolar. 


\section{El significado de la historia para los jóvenes chilenos}

Existen estudios descriptivos que han intentado caracterizar las ideas de los estudiantes concerniente a la historia en un momento dado en el tiempo (Barton 2010). En ocasiones, algunos de estos estudios muestran cómo las personas asumen con frecuencia que la historia es una cuestión de "sentido común" carente de las abstracciones que se encuentran en disciplinas como las matemáticas o las ciencias (Lee 2006). Por lo mismo, en esta investigación quisimos indagar una primera cuestión general referida a la relación que establecen los jóvenes chilenos con la historia. Los estudiantes de segundo año de Educación Media que participaron de la muestra le asignan importancia y valoran positivamente el conocimiento de la historia (véase Figura 1). Las afirmaciones con las que declaran estar más de acuerdo son aquellas que conciben la historia como un tipo de conocimiento que muestra lo que está por detrás de la manera de vivir en el presente y explica los problemas actuales, en segundo lugar conciben la historia como una forma de entender nuestras vidas como parte de los cambios que se producen con el paso del tiempo, y, por último, valoran la historia como una posibilidad de aprender con los errores y aciertos de los otros. Es decir, para los estudiantes, la historia no es una simple asignatura que entrega contenidos descontextualizados, muchas veces alejados de la realidad y del tiempo presente, sino que les resulta una herramienta útil para aproximarse a explicaciones referidas a su día a día. Complementariamente, esta apreciación aparece con mayor claridad cuando revisamos las opciones menos aceptadas por los jóvenes, es decir, aquellas en las que los estudiantes manifestaron mayor desacuerdo. Por un lado, no comparten la idea de que la historia sea algo que ya pasó y murió y que nada tiene que ver con su vida y, por otro lado, tampoco les parece que la historia pueda considerarse una materia de la escuela y nada más. Este conjunto de ideas generales nos ofrece, a simple vista, una primera escena generosa y una conexión positiva con la disciplina histórica aflorando una cierta flexibilidad en el pensamiento histórico de los jóvenes que les permite conectar sus preocupaciones personales (su vida) con una temporalidad mayor, particularmente con el tiempo presente. Como señalan otras investigaciones, los estudiantes se definen a sí mismos permanentemente "como interesados en la historia y conscientes de su importancia" (Barton 2010:109). Las preferencias de los estudiantes le otorgan valor al estudio formal de la disciplina histórica, pero ven este conocimiento como algo que excede los formalismos del conocimiento escolarizado. De algún modo, lo que se lograría por medio de la historia se aproxima a una cierta comprensión de la actualidad, de la propia experiencia histórica y, por tanto, colaboraría en la conflictiva constitución de identidades en el mundo actual.

Schmidt (2005) recogió evidencia del pensamiento histórico de los jóvenes brasileños de la

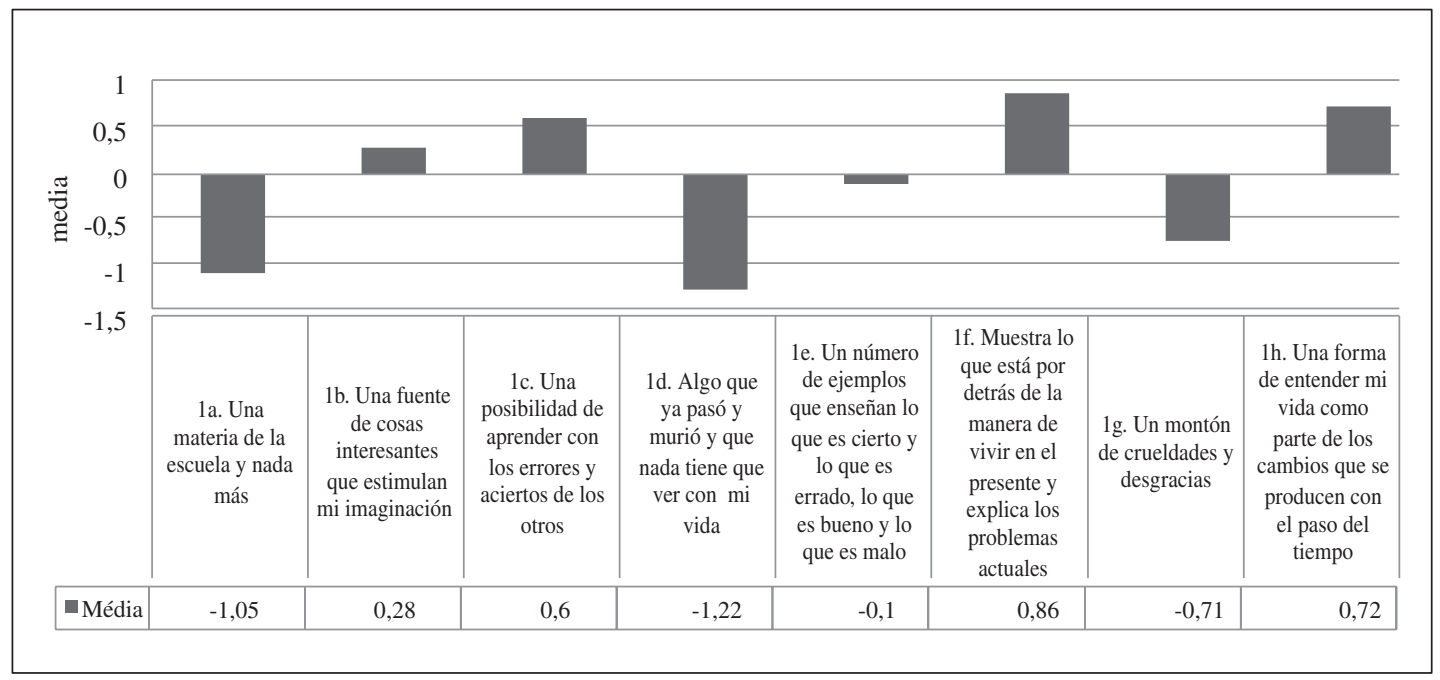

Figura 1. ¿Qué significa la historia para ti? 
ciudad de Curitiba y concluyó que un porcentaje muy alto de esos estudiantes rechaza la idea referida a que la historia sea una asignatura escolar y nada más, coincidiendo con lo que piensan los jóvenes de nuestro estudio. Es decir, en ambos casos se valoró la historia como aprendizaje e interpretación de la realidad. La autora pudo comparar las respuestas de los jóvenes brasileños con datos disponibles de jóvenes europeos, encontrando, en este caso, tanto valoraciones similares como dispares. Los adolescentes europeos y brasileños no creen que el conocimiento histórico sea algo negativo, relacionado solo con el pasado y con acontecimientos vinculados con la muerte, la crueldad o las catástrofes. Sin embargo, mientras los jóvenes brasileños valoran la historia como un medio para contemplar su propia vida como parte de la evolución histórica, esta idea es relevante solo para algunos jóvenes de Europa (portugueses, israelíes, árabes y palestinos).

Un segundo tipo de valoraciones se pueden observar en la Figura 2, donde los estudiantes respondieron a la pregunta ¿cuál es la importancia de cada uno de los objetivos en el estudio de la historia? Frente a esta interrogante los estudiantes disponían de tres opciones (a) Conocer el pasado, (b) Comprender el presente, (c) Buscar orientación para el futuro. Las afirmaciones que concentraron mayor acuerdo entre los jóvenes son las que señalan que la historia sirve para conocer el pasado y para comprender el presente. Luego, también con una valoración positiva, se ubicó la idea que sostiene que la historia permite buscar orientación para el futuro, en promedio menor que las anteriores pero ampliamente aceptada por los estudiantes. Estos datos parecen complementarse con lo expuesto en la Figura 1, confirmando ciertos aspectos del análisis. Para los estudiantes, el estudio de la disciplina apunta fundamentalmente al conocimiento del pasado, pero como vimos se trataría de un pasado estrechamente vinculado al presente. Aunque la comprensión de lo que sucede en la actualidad podría concentrar los intereses históricos de los jóvenes, no resulta nada despreciable el reconocimiento que hacen a la función de orientación temporal que debiera caracterizar al estudio de la historia. Recordemos que según J. Rüsen (1992; 2004), la historia es un nexo significativo que entrelaza el pasado, el presente y el futuro, y la conciencia histórica tiene, ante todo, una función práctica que es la de orientación temporal. Es por medio de estos procesos que una persona se hace parte de un todo temporal más extenso que el de su vida individual. Reconociendo con claridad las tres dimensiones temporales con las que se vincula la historia, los jóvenes muestran el potencial formativo de estos procesos de pensamiento (Rüsen 2010), pues, en tanto actos mentales propios del desarrollo de la conciencia histórica, nos revelarían "el tejido del cambio temporal dentro del cual están atrapadas nuestras vidas y las perspectivas futuras hacia las cuales se dirige el cambio" (Rüsen 1992: 29).

Ahora bien, investigaciones similares en jóvenes de Brasil, Argentina y Uruguay muestran una alta probabilidad de que la enseñanza de la Historia sea

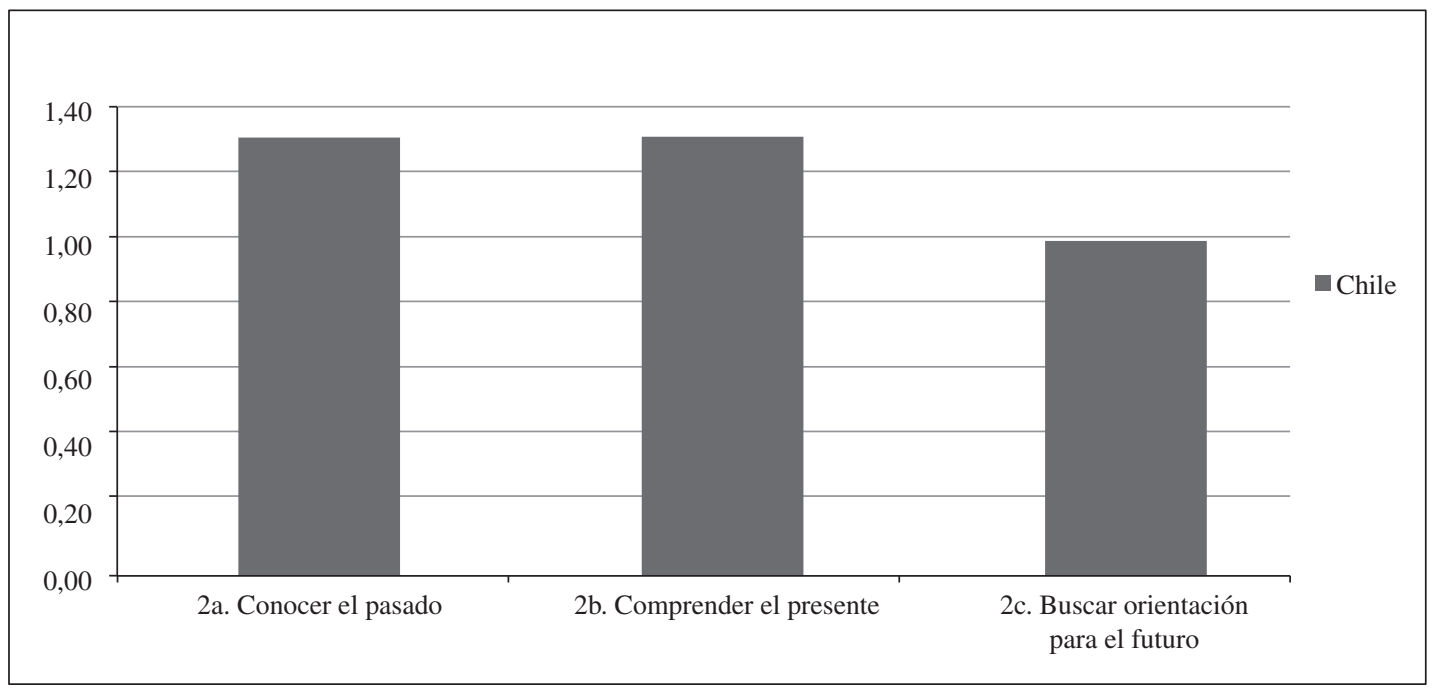

Figura 2. En tu opinión, ¿cuál es la importancia de cada uno de los siguientes objetivos en el estudio de la historia? 
valorizada como significativa por el alumnado, lo que no expresa necesariamente que sea de su agrado. Este tipo de datos mostraría que el discurso escolar, en específico la Historia como disciplina, ha sido bien absorbido por los alumnos, cuestión que se manifiesta en una reproducción de los discursos acerca de la importancia de sus objetivos (Cerri y Amézola, 2010). Los autores identifican que entre los jóvenes brasileros, a diferencia de lo que muestran los datos recogidos en nuestro estudio, se percibe una tendencia a una apropiación pragmática, proyectiva y propositiva del conocimiento histórico, con el foco de atención dirigido al futuro.

\section{El lugar de los materiales y recursos en el aprendizaje histórico}

Sin duda, uno de los aspectos que mayor incidencia tiene en la lejanía o proximidad que puedan desarrollar los jóvenes hacia la historia, en tanto saber escolar, está asociado a aquellos recursos o materiales que protagonizan los procesos de mediación didáctica. La Figura 3 muestra cuáles serían los materiales educativos utilizados en la escuela para el estudio de la historia que resultan de mayor agrado para los estudiantes. Puede apreciarse que entre las opciones más valoradas se encuentra aquello que podría definirse como trabajo extraaula, vinculado a visitas a museos o acceso directo a lugares con valor histórico (sitos patrimoniales), en segundo lugar el aprendizaje por medio de películas, así como también las explicaciones de los profesores y los documentales de la televisión. Aunque frente a esta cuestión no hay percepciones negativas, el recurso que muestra un menor indicador de agrado es, tal vez, el más utilizado en las salas de clases en Chile: el texto escolar. El efecto de los manuales escolares o de los textos didácticos en el aprendizaje histórico de los niños de la escuela primaria ya ha sido estudiado, mostrando una deformación del conocimiento histórico que se convierte, en esos niveles, en una historia única y verdadera sancionada en el libro (Lee 2006).

En relación con este aspecto, K. Barton (2010:103) ha dado cuenta que la investigación internacional muestra frecuentemente que "los estudiantes también encuentran los textos de los libros de divulgación más interesantes, informativos y entretenidos que los textos tradicionales". De igual modo, es sabido que el uso de materiales distintos $\mathrm{o}$ adicionales al texto de estudio también se asocia con notas más altas en las pruebas de rendimiento de la asignatura en cuestión (Barton, K. 2010), ya que genera en cierta medida una mayor motivación para los estudiantes al momento del aprendizaje.

R. Cuesta (1998) ya se encargó de mostrarnos, para el caso español, la existencia de trabajos analíticos e informes que dan cuenta del predominio y la perdurabilidad de ciertos usos educativos de la historia marcadamente tradicionales, que tienen en la explicación del profesor y en el libro de texto sus figuras centrales. Mediante este tipo de presentación de los contenidos históricos como forma de conocimiento tópico (Schmidt 205), asociados al

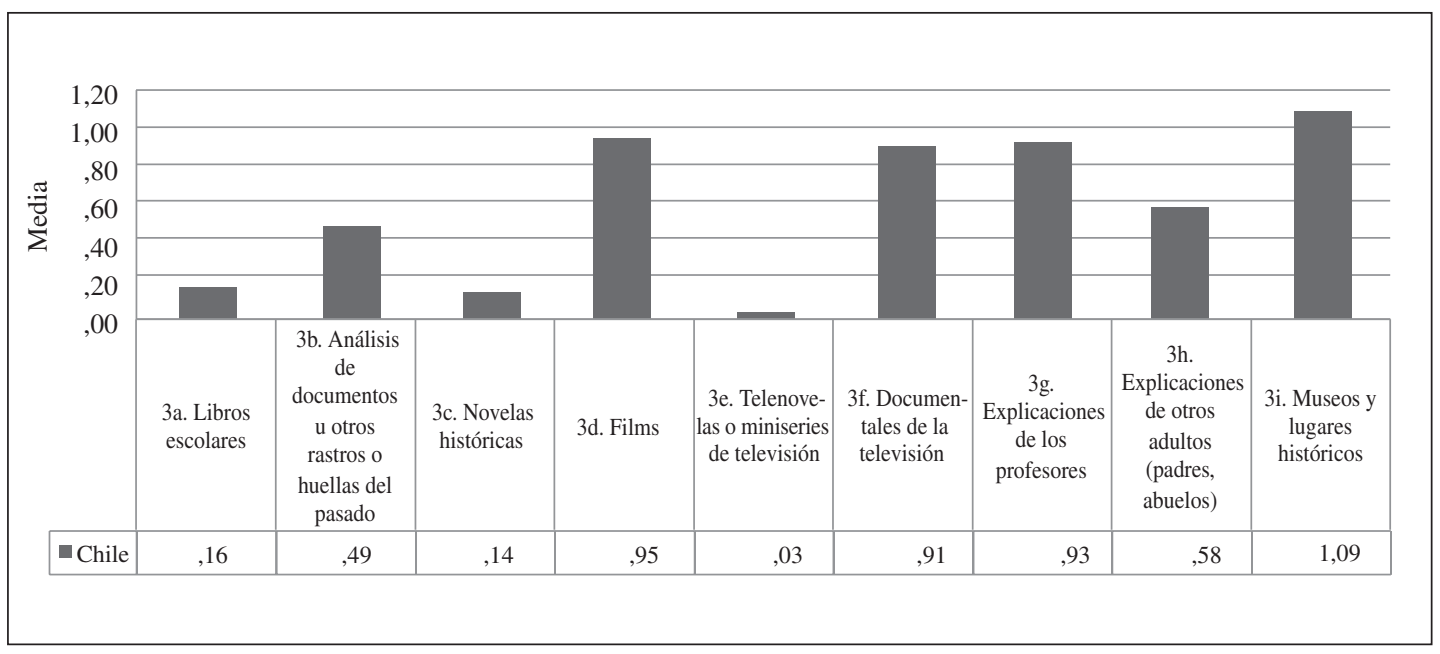

Figura 3.¿Qué materiales y recursos de la historia utilizadas en la escuela resultan más de tu agrado? 
desempeño del común de los docentes en la disciplina histórica, podemos evidenciar que las clases en las escuelas siguen siendo de carácter expositivo. De acuerdo con este argumento, "quizá sea posible hallar, por encima de las divergencias ideológicas e historiográficas, un sustrato común, una ideología profesional de la vida cotidiana y una doxa mayoritarias que explicarían algunas de la prácticas más extendidas de la enseñanza de la historia" (Cuesta 1998: 175). Convengamos, en todo caso, que estas rutinas o cualquier otro hábito escolar no son fruto de ninguna invención caprichosa, sino más bien "obedecen a una lógica social de producción de significados" (Cuesta 1998:189).

La constatación anterior puede tornarse importante si se considera que la explicación del profesor se encuentra dentro de los materiales más valorados por los estudiantes chilenos para aprender historia. Obviamente, este arraigado tipo de método debiera encontrar un complemento equilibrado en aquellos otros recursos que por fuera del aula o desde dentro de las culturas juveniles presionan por modificar las prácticas más tradicionales. Directamente asociada a la pregunta anterior se pidió también a los estudiantes pronunciarse por aquellos recursos o materiales que resultaban más confiables en el estudio de la historia (véase Figura 4). Frente a esto, los jóvenes manifestaron abierta desconfianza hacia las telenovelas, miniseries y películas. Es decir, catalogaron dentro de lo menos confiable aquellas formas de divulgación cuyo romance con la ficción histórica pudiera ser más criticable. Pese a ser recursos que resultan del agrado de los estudiantes, no logran ganarse aún el crédito de sus receptores probablemente por su origen ya sea comercial, propagandístico o de simple entretenimiento. La imagen aún poderosa de un conocimiento histórico formalizado y anclado a las viejas instituciones del saber hace que las producciones mediáticas gocen de poco prestigio en el mundo escolar: entretienen en lugar de enseñar; dramatizan en lugar de contextualizar; o, peor aún, humanizan en lugar de objetivar. Tal vez eso sea lo que opinen los estudiantes una vez que se ha consolidado en ellos la imagen de la historia como un saber monolítico, neutral y escasamente narrativo.

Si bien las producciones cinematográficas no pretenden ser un fiel reflejo de los hechos históricos ocurridos a lo largo del tiempo, cuestión que es reconocida con claridad por los estudiantes en los bajos porcentajes de aceptación, sí podría ser un recurso utilizado por parte de los docentes para tensionar el análisis de ciertas materias o con el fin de promover el razonamiento moral y tomar decisiones considerando diversos cursos de acción posibles (Rüsen 2004). De esta manera, desarrollar competencias propias de la conciencia histórica como la competencia narrativa difícilmente logre movilizarse con la sola exposición del profesor. Dentro de las opciones más confiables aparecen los museos o lugares históricos, las explicaciones de profesores y los documentales de televisión. De estos resultados

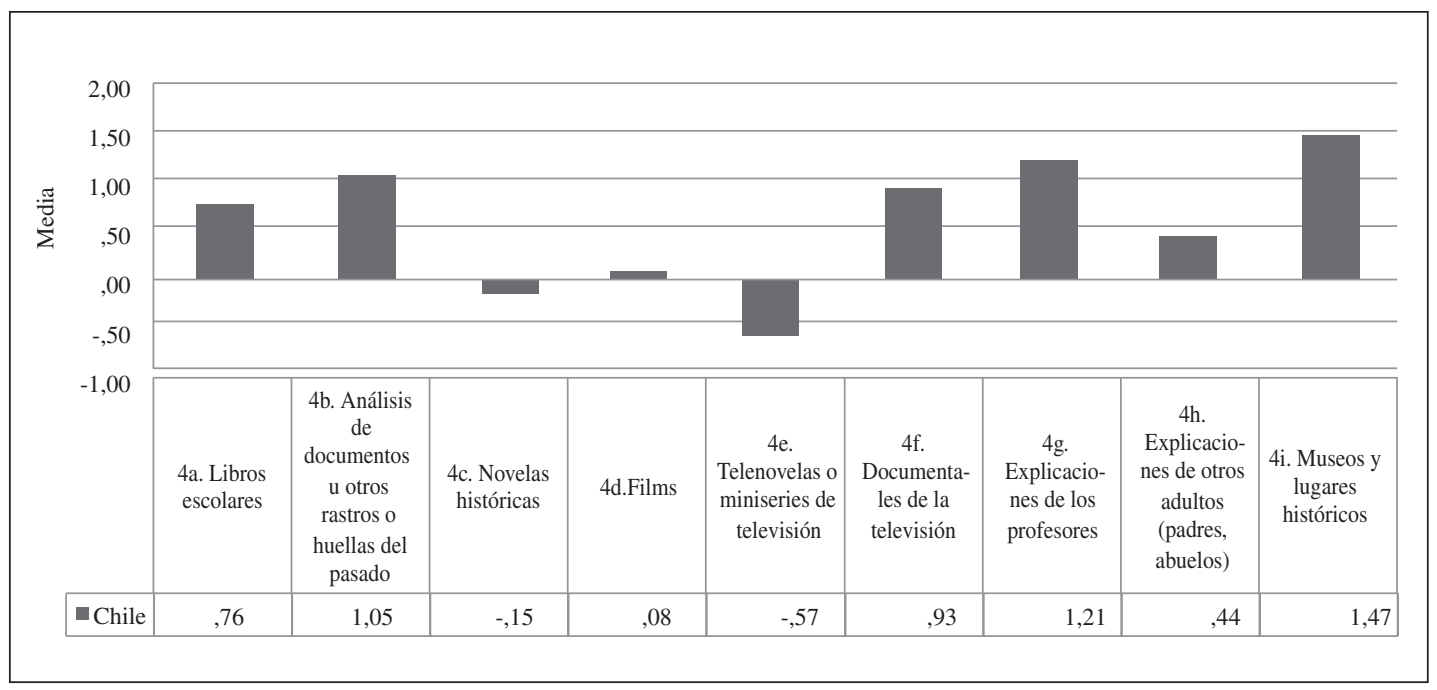

Figura 4. ¿En qué materiales y recursos utilizados en la historia confías más? 
hay valiosos elementos que podrían ser tomados en cuenta para abrir el espectro de recursos utilizados en las escuelas para la enseñanza de la historia y de esta manera motivar a los estudiantes a aprender acerca de la disciplina desde un enfoque diferente. Por ahora, la investigación internacional muestra escasos avances en relación con el uso de materiales menos tradicionales para aprender historia. Según sistematizaciones recientes:

"se sabe muy poco sobre cómo niños y adolescentes captan el pasado en contextos que sean menos formales o escolares, es decir, escuchando las historias familiares, mirando documentales en la televisión, discutiendo de política con iguales, mirando una exposición de un museo o asistiendo a un acto conmemorativo" (Barton 2010:109)

En cualquier caso, los estudiantes que participaron de nuestro estudio coinciden con adolescentes de otros contextos en el sentido que dicen haber aprendido acerca del pasado de sus padres, hermanos u otros familiares; a partir de medios de comunicación como la televisión, películas, libros de divulgación; o en menor medida, por visitas a museos y sitios históricos (Barton 2010). Se comprenderá, entonces, que la historia como conocimiento escolar es, pues, el resultado de una práctica social negociada entre sus principales protagonistas (docentes y estudiantes) y un conjunto de determinaciones sociales del que no tienen mayor control (Cuesta 1998).

\section{Regularidades, tradiciones y desilusiones en la clase de Historia}

En la Figura 5 se muestran las percepciones de los estudiantes en relación con la pregunta ¿qué ocurre normalmente en las clases de historia?, frente a esta interrogante los estudiantes plantearon que dentro de las cosas más comunes que suceden está el uso de libros escolares o algún otro material impreso, en segundo lugar escuchan las explicaciones de los profesores relativos al pasado, y en tercer término son informados de lo que fue bueno o malo, verdadero o falso en la historia. De acuerdo con estas opciones, que son las que reúnen mayor aceptación entre los estudiantes que respondieron el cuestionario, podemos reafirmar lo expuesto en puntos anteriores: las clases de historia siguen teniendo un enfoque tradicional, en donde el profesor es quien hace la clase, apoyándose apenas en el texto de estudio. Es él quien porta la verdad, ya que es quien decide según lo revelado por los estudiantes, qué es lo bueno/malo o verdadero/ falso de la historia, sin posibilitar la exploración, asimilación y reflexión de los estudiantes respecto de los contenidos que se le plantean. Como ha sido planteado por investigadores de otros continentes

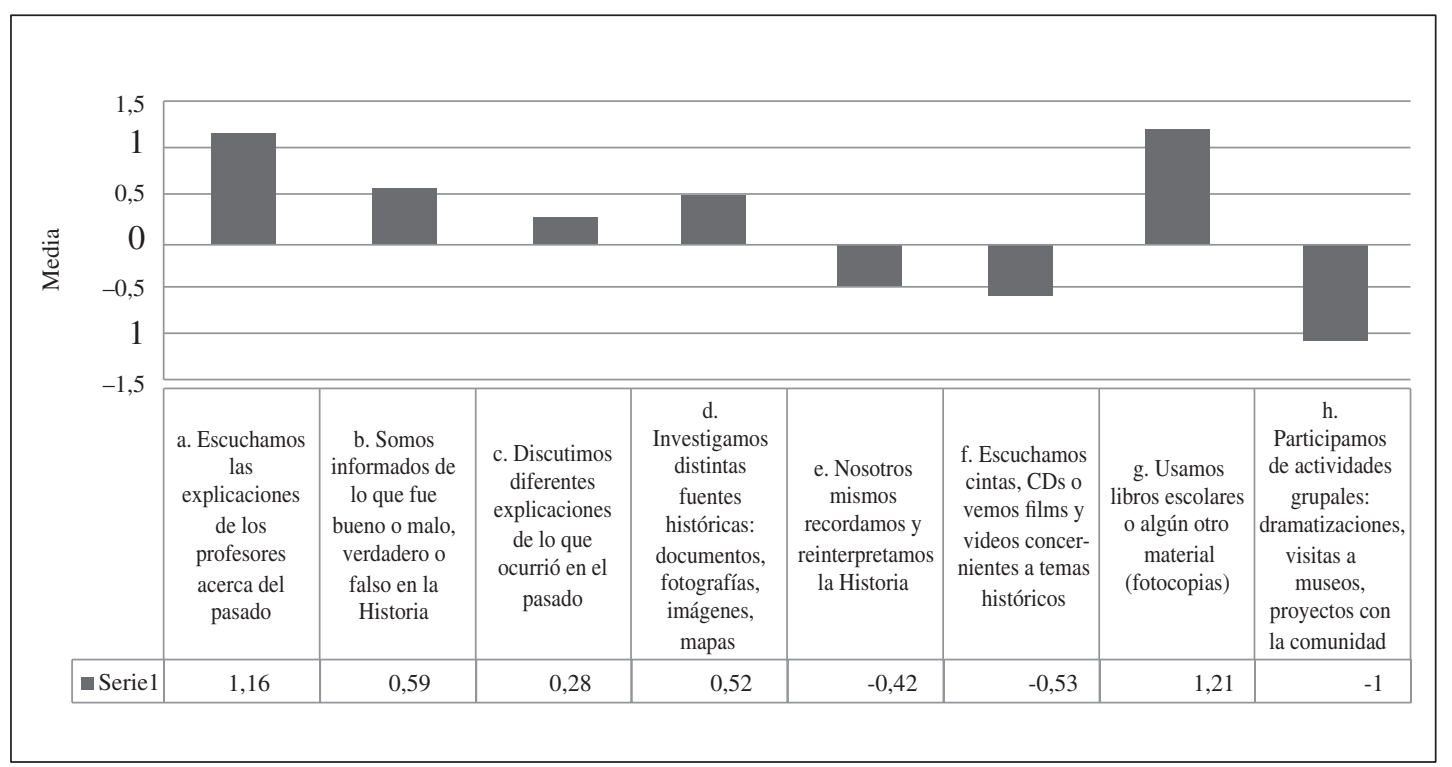

Figura 5. ¿Qué ocurre normalmente en las clases de historia? 
(Tutiaux-Guillon 2006), en la enseñanza de la historia en el nivel secundario priman los conocimientos factuales o nocionales, textos y documentos rara vez se someten a la crítica y su credibilidad como vectores de conocimiento casi nunca se pone en duda, terminando como sustitos de lo real.

Del mismo modo, aquella búsqueda imposible de lo verdadero y lo falso en la historia, de lo bueno y lo malo, nos da una clara señal de la idea de Historia que se ofrece en aquellas aulas: ejemplar, heroica, monumental. Si entendemos que el objetivo de las prácticas de enseñanza debiera apuntar a modificar las ideas previas de los estudiantes, permitiendo de esta manera su reformulación (Carretero et al. 2013), y si consideramos el aprendizaje histórico como un cambio estructural de la conciencia histórica (Rüsen 1992), las rutinas de enseñanza retratadas por los estudiantes se ubican muy lejos de tales propósitos. $\mathrm{Al}$ parecer, lo que se ha constatado en otras investigaciones vuelve a reafirmarse para el caso de los jóvenes chilenos. Es frecuente que los estudiantes, en sus clases de historia, se sientan "sobrepasados y frustrados por la cantidad de papel impreso que tienen que afrontar" (Barton 2010:101).

Tal vez lo único que marque un tipo de práctica diferenciada, según lo que perciben los estudiantes, es el reconocimiento de la investigación y el uso de fuentes históricas como una de las actividades que tienen cierta presencia en el aula de historia. Esta metodología, pese a que pudiera estar habitada por las mismas viejas rutinas que se asocian al profesor expositivo, constituye una ventana que posibilita una mirada distinta al ejercicio de la enseñanza. En primer lugar, este tipo de prácticas vincula a los estudiantes directamente con los métodos históricos; y, por otro lado, la aproximación que hacen los estudiantes a los diversos tipos de fuentes los faculta para desarrollar la competencia narrativa y elaborar relato histórico. Para que estas cuestiones prosperen será preciso, antes, desmontar aquellas preconcepciones o representaciones que llevan a muchos estudiantes a entender las fuentes históricas como fuentes de información directa, objetiva e irrefutable respecto del pasado (Barton 2010). Siguiendo el argumento de P. Lee (2006), si muchos alumnos ven todavía el pasado como algo permanente es porque la pregunta acerca de cómo conocemos en historia, simplemente no ha surgido.

En relación con las actividades menos frecuentes en las clases de historia los estudiantes señalan haber tenido muy pocas oportunidades de escuchar cintas, $C D$ o ver films y videos de temas históricos, y tampoco es habitual que sean ellos mismos quienes recuerden o reinterpreten la historia. Es evidente el hecho de que estas actividades no sean promovidas en el aula va en directa relación con lo sugerido con anterioridad, la innovación y la experimentación no son solo acciones que se conjuguen habitualmente con el verbo enseñar (historia). Los docentes de historia no utilizan estrategias o materiales que permitan que los estudiantes puedan desarrollar otras dimensiones de la conciencia histórica como la dimensión estética o política; los niveles de concreción y abstracción aparecen descompensados predominando lo primero por sobre lo segundo; y en las definiciones metodológicas se privilegian aquellas desgastadas prácticas que favorecen la lógica emisor-receptor tan clásica de la escolarización del siglo XX y que recuerda de manera permanente la educación bancaria denunciada por Freire.

Finalmente, se solicitó a los estudiantes que respondieran a la pregunta ¿en qué se concentran más sus clases de historia? (ver Figura 6). Dentro de las proposiciones que encontraron mayor reconocimiento entre los estudiantes destacan afirmaciones como: buscamos conocer los principales hechos de la historia; intentamos entender cómo era la vida en el pasado teniendo en cuenta todos los puntos de vista; y, empleamos la historia para entender la situación del mundo actual y descubrir las tendencias de cambio. Por otro lado, la afirmación frente a la que las perspectivas de los estudiantes se encuentran más divididas es la que señala que las clases de historia se centran en estudiar en una forma que resulta interesante e incentiva la imaginación.

De acuerdo con Lee (2006), muchos alumnos piensan el pasado como si fuera un paisaje lejano detrás de nosotros, fuera de nuestro alcance, fijo y eterno. En ese paisaje solo sería reconocible cierto tipo de relieve que por su dimensión o envergadura resulta de una invisibilización injustificada. Ese relieve invariable lo constituyen los hechos de la historia, diríamos, aquello que no se puede dejar de ver. La dificultad de esta perspectiva es que la historia escolar termina convirtiéndose en una sucesión de eventos y acciones localizadas muy específicamente en el tiempo y en el espacio que no otorgan sentido de continuidad o de ruptura. Cuando los estudiantes chilenos señalan que sus clases se concentran en conocer los principales hechos de 


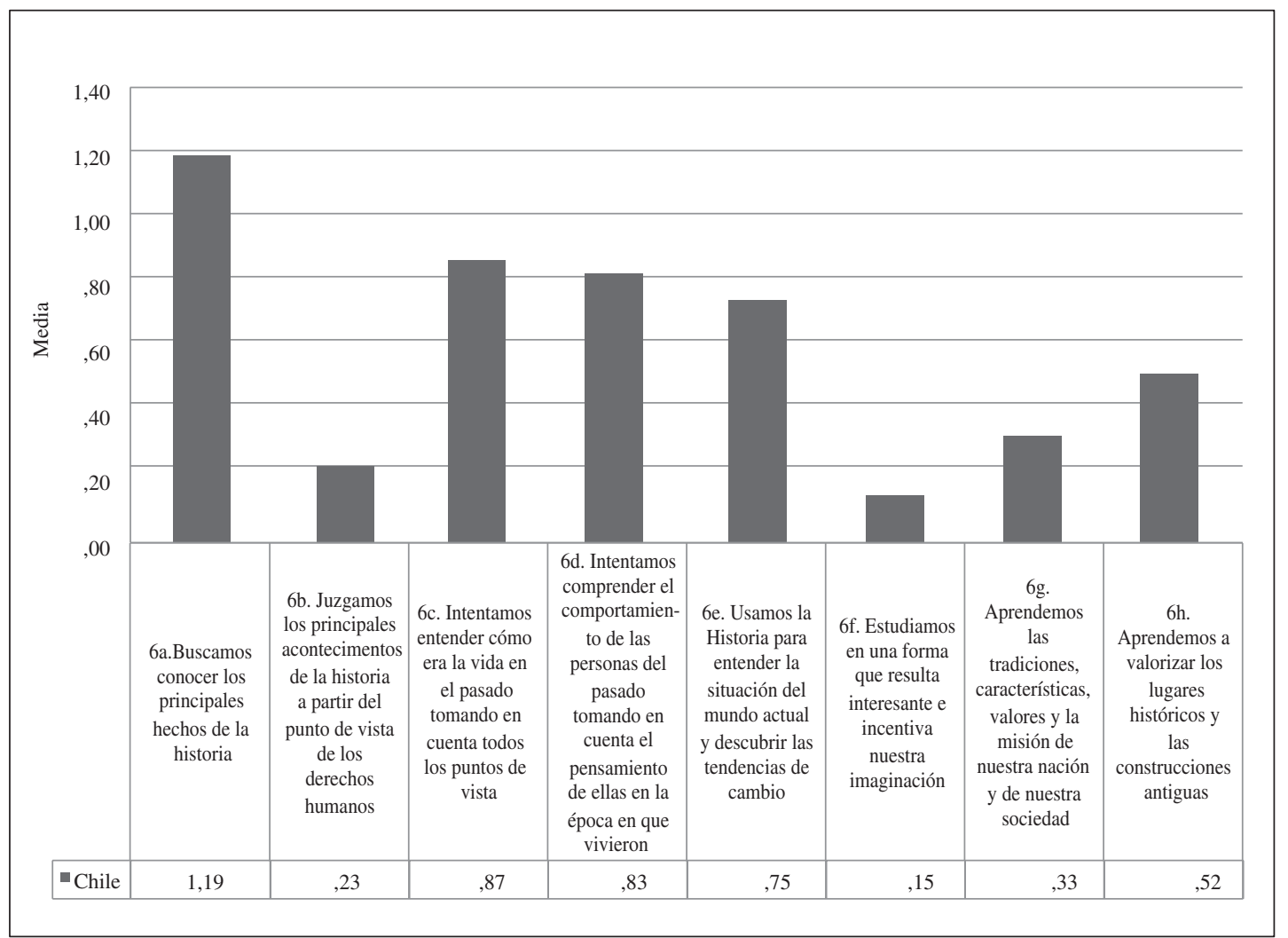

Figura 6. ¿En qué se concentran más tus clases de historia?

la historia están haciendo alusión, probablemente, a esa lógica.

En cuanto a la idea de conocer la vida en el pasado, es relevante que los estudiantes pongan su atención en esta proposición, ya que pone el acento en la consideración de diversos puntos de vista en el estudio histórico. Tal enfoque permitiría reflexionar de manera más crítica en relación con las experiencias del pasado, a la toma de decisiones, y desarrollar la empatía en los estudiantes al cuestionar razonamientos ajenos sin perder de vista la temporalidad de los marcos de acción.

Como podemos apreciar, las tres actividades relacionadas con la historia en las aulas más reconocidas por los estudiantes son coherentes con lo que se aprecia en la Figura 2 comentada más arriba. Las tres dimensiones temporales (pasado, presente y futuro) se encuentran implícitas a la hora de pensar en qué cuestiones se concentran las clases de historia, en definitiva se trata de: conocer el pasado, entender el presente y, en menor medida, hacerse la pregunta por el cambio histórico. Ciertamente, si pudiéramos avanzar en la comprensión de las nociones que los jóvenes elaboran en relación con el cambio, la orientación temporal o la identidad daríamos pasos fundamentales en la búsqueda de nuevos rumbos para la enseñanza de la historia (Barca 2013).

\section{Reflexiones finales}

El rotundo impacto del desarrollo tecnológico, el individualismo y las redes, el compromiso con las cuestiones medioambientales o la crisis de expectativas a nivel nacional, dieron forma a una generación de jóvenes del siglo XXI cuyo lugar histórico es radicalmente diferente al de todos sus predecesores.

La manera intuitiva en que los jóvenes intentan dar respuestas a los requerimientos y pulsiones temporales que les impone la sociedad contemporánea resulta ineficaz o claramente insuficiente. Las estrategias de aprendizaje histórico derivadas del modelo escolar resultan igualmente deficientes 
considerando las necesidades de orientación que se derivan de un mundo interconectado y diverso. Las clases de historia que se bosquejan a partir de las percepciones estudiantiles comentadas en este trabajo describen un formato conservador, centrado en la acción del docente y en los dispositivos escritos con que cuente, con escasa investigación autónoma por parte de los estudiantes donde la palabra del profesor sigue siendo autoridad y seguramente en no pocos casos verdad absoluta.

Es evidente que existe una resistencia a la innovación y un apego a prácticas de enseñanza positivistas. Sin embargo, no se trata de algo tan sencillo como renovar a los profesores de historia, pues los estudiantes expresan en sus percepciones acerca de la historia una estructura igualmente positivista: desechan los soportes tecnológicos, se aferran a la confiabilidad que depositan en quien les enseña, y reconocen en lo escrito una fuente de autoridad que no le asignan a ningún otro recurso de aprendizaje. Si se quiere desarrollar en los estudiantes habilidades distintas a las tradicionales, como aquellas derivadas del desarrollo de la conciencia histórica, se precisa, ante todo, indagar en las ideas que los estudiantes elaboran tanto dentro de los contextos escolares como en las dinámicas de socialización extraescolar y explicar las razones de tales representaciones. Según señala N. Tutiaux-Guillon (2003), adquirir una conciencia histórica implica ser consciente de que el pasado participa del presente dejando su huella.
Usar esta perspectiva histórica para mirar el mundo y la experiencia individual o colectiva supone hacer referencias históricas, razonamientos históricos y actuaciones. Estimar que una herramienta así es pertinente obliga a explorar las relaciones entre pasado, presente y futuro en la enseñanza.

$\mathrm{Si}$ los estudiantes estuvieran equipados con algunas herramientas intelectuales básicas del pensamiento histórico, provistas desde la clase de historia, estarían en condiciones de tener una visión global respecto de su presente y su horizonte futuro, y así poder desafiar todas aquellas interpretaciones del pasado colectivo que enmascaran viejas liturgias de sometimiento y resignación.

\section{Agradecimientos}

Los autores agradecen a la Dirección de Investigación de la Universidad Academia de Humanismo Cristiano (UAHC) por el financiamiento obtenido por este Núcleo Temático de Investigación (NTI) durante los años 2012 y 2014 que han hecho posible su ejecución y permanencia. Agradecemos también a la carrera de Pedagogía en Historia y Ciencias Sociales de la UAHC permitir el desarrollo de esta línea de investigación didáctica. Finalmente nuestra permanente gratitud al Dr. Luis F. Cerri (UEPG, Brasil) por su apoyo desinteresado y generoso al desarrollo de este tipo de discusiones en el Cono Sur.

\section{Referencias Citadas}

Barca, I.

2013 Conciencia histórica: pasado y presente en la perspectiva de los jóvenes en Portugal. Clío y Asociados 17:16-26

Barton, K.

2010 Investigación sobre las ideas de los estudiantes acerca de la historia. Enseñanza de las Ciencias Sociales 9:97-114.

Bergmann, K.

1990 A Historia na Reflexao Didática. Revista Brasileira de História. Vol. 9 No 19: 29-42 Sao Paulo

Carretero, M., Castorina, J. A., Sarti, M., Van Alphen, F. y

Barreiro, A.

2013 La Construcción del conocimiento histórico. Propuesta Educativa No 39 Vol. 1: 13-23.

Cerri, L. F.

2011 Ensino de história e consciencia histórica. Implicacoes didaticas de uma discussao contemporánea. Río de Janeiro, Editora FGV.

Citron, $\mathrm{S}$.

1982 La historia y las tres memorias. En La Historia en el aula, compilado por M. Pereyra, pp. 113-124. Instituto de Ciencias de la Educación, Universidad de La Laguna, España.
Cuesta, R.

1998 Clio en las Aulas. La enseñanza de la Historia en España entre reformas, ilusiones y rutinas. Akal, Madrid, España.

Dayrell, J.

2007 A escola "faz" as juventudes? Reflexões em torno da socialização juvenil. Educação e Sociedade Vol. 28, $\mathrm{N}^{\circ}$ 100: 1105-1128, Campinas, Brasil.

Feixa, C.

2006 Generación XX. Teorías sobre la juventud en la era contemporánea. Revista Latinoamericana de Ciencias Sociales, Niñez y Juventud Vol. 4, $\mathrm{N}^{\circ}$ 2: 21-45.

Garcés, M.

2012 El despertar de la sociedad: Movimientos sociales en América Latina y Chile. Editorial LOM, Santiago, Chile.

González, F.

2012 Reforma educativa en la postdictadura chilena: multitudes, mercado y protesta social. ConCiencia Social, $\mathrm{N}^{\circ}$ 16: 159-167.

Giroux, $\mathrm{H}$.

1994 Jóvenes, diferencia y educación postmoderna. En Nuevas Perspectivas Críticas en Educación, M. Castells, R. 
Flecha, P. Freire, H. Giroux, D. Macedo, P. Willis, pp. 97128. Paidós Ibérica, Madrid.

Lee, $\mathrm{P}$.

2006 Em direção a um conceito de literacia histórica. Educar número especial: 131-150, Universidade Federal do Paraná, Curitiba.

Martín-Barbero, J.

1998 Jóvenes: desorden cultural y palimpsestos de identidad. En Viviendo a toda. Jóvenes, territorios culturales y nuevas sensibilidades, Editado por Universidad Central-DIUC, pp. 22-37, Siglo del Hombre, Santa Fe de Bogotá.

Pagés, J.

2015 Saberes históricos construidos -saberes históricos apropiados. Una reflexión desde la didáctica de la historia. En Memória, sensibilidades e saberes, organizado por E. Zamboni, M. C. Galzerani, C. Pacievitch, pp. 304-325. Editora Alínea, São Paulo, Brasil.

Reguillo, R.

2000 Emergencia de Culturas Juveniles. Norma, Bogotá, Colombia.

Rüsen, J.

1992 El desarrollo de la competencia narrativa en el aprendizaje histórico. Una hipótesis ontogenética relativa a la conciencia moral. Traducido por S. Finocchio. Propuesta Educativa Año 4 N $^{\circ}$ 7: pp. 27-36

Rüsen, J.

2004 Historical Consciousness: Narrative Structure, Moral function, and ontogenetic development. En Theorizing Historical Consciousness, editado por P. Seixas, pp. 63-85. University of Toronto Press, Toronto, Canada.

Rüsen, J.

2010 Aprendizado historico. En Jörn Rüsen e o Ensino de História, organizado por M. A. Shmidt, I. Barca, E. Martins, pp. 41-49. Editora UFPR, Curitiba, Brasil.
Salazar, G.

2003 La historia desde abajo y desde dentro. Facultad de Artes Universidad de Chile, Santiago.

Salazar, G. y Pinto, J.

2002 Historia Contemporánea de Chile. Tomo V: Niñez y Juventud. Editorial LOM, Santiago.

Santisteban, A. y Anguera C.

2014 Formación de la conciencia histórica y educación para el futuro. Clío y Asociados $\mathrm{N}^{\circ}$ 19: 249-267

Schmidt, M. A.

2005 Jóvenes brasileños y europeos: identidad, cultura y enseñanza de la historia (1998-2000). Enseñanza de las Ciencias Sociales, 4, 53-64.

Shmidt, M. A. y Cainelli, M.

2015 A consciência Histórica como o lugar e o propósito da aprendizagem histórica. Introdução a um diálogo com a teoria Jörn Rüsen. En Memória, Sensibilidades e Saberes, organizado por E. Zamboni, M. C. Galzerani, C. Pacievitch, pp. 116-128. Editora Alínea, São Paulo, Brasil.

Sposito, M.

1999 Juventude: crisis, identidad y escuela. En Múltiplos Olhares sobre Educação e Cultura, Organizado por J. Dayrell, pp. 96-104. Editora UFMG, Belo Horizonte, Brasil. Tutiaux-Guillon, N.

2003 Los fundamentos de una investigación sobre la concepción de las finalidades cívicas y culturales del profesorado de geografia e historia. Objetivo de esta etapa. Enseñanza de las Ciencias Sociales, 2, 27-35

Tutiaux-Guillon, N.

2006 La investigación sobre la enseñanza y el aprendizaje de las ciencias sociales en las aulas. El ejemplo francés. En Formar para investigar, investigar para formar en Ciencias Sociales. A. Goméz Rodríguez y M. P. Núñez Galeano, pp. 19-38. Asociación Universitaria del Profesorado de Didáctica de las Ciencias Sociales. Málaga, España. 
